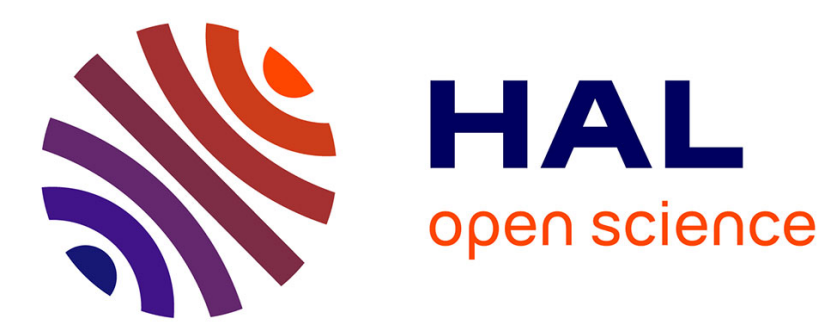

\title{
L'approche ressources et compétences comme clé de lecture du processus d'élaboration d'une ressource originale : la marque territoire \\ Corinne Rochette
}

\section{- To cite this version:}

Corinne Rochette. L'approche ressources et compétences comme clé de lecture du processus d'élaboration d'une ressource originale : la marque territoire. Gestion et management public [2012-..], $2012,1 / \mathrm{n}^{\circ} 1$ (1), 10.3917/gmp.001.0004 . hal-01734716

\author{
HAL Id: hal-01734716 \\ https://hal.uca.fr/hal-01734716
}

Submitted on 14 Mar 2018

HAL is a multi-disciplinary open access archive for the deposit and dissemination of scientific research documents, whether they are published or not. The documents may come from teaching and research institutions in France or abroad, or from public or private research centers.
L'archive ouverte pluridisciplinaire HAL, est destinée au dépôt et à la diffusion de documents scientifiques de niveau recherche, publiés ou non, émanant des établissements d'enseignement et de recherche français ou étrangers, des laboratoires publics ou privés. 


\section{L'APPROCHE RESSOURCES ET COMPÉTENCES COMME CLÉ DE LECTURE DU PROCESSUS D'ÉLABORATION D'UNE RESSOURCE ORIGINALE : LA MARQUE TERRITOIRE}

Corinne Rochette

AIRMAP | «Gestion et management public »

2012/1 Volume $1 / \mathrm{n}^{\circ} 1 \mid$ pages 4 à 20

Article disponible en ligne à l'adresse :

https://www.cairn.info/revue-gestion-et-management-public-2012-1-page-4.htm

\section{Pour citer cet article :}

Corinne Rochette, «L'approche ressources et compétences comme clé de lecture du processus d'élaboration d'une ressource originale : la marque territoire », Gestion et management public 2012/1 (Volume 1/n¹), p. 4-20.

DOI 10.3917/gmp.001.0004

Distribution électronique Cairn.info pour AIRMAP .

(C) AIRMAP. Tous droits réservés pour tous pays.

La reproduction ou représentation de cet article, notamment par photocopie, n'est autorisée que dans les limites des conditions générales d'utilisation du site ou, le cas échéant, des conditions générales de la licence souscrite par votre établissement. Toute autre reproduction ou représentation, en tout ou partie, sous quelque forme et de quelque manière que ce soit, est interdite sauf accord préalable et écrit de l'éditeur, en dehors des cas prévus par la législation en vigueur en France. Il est précisé que son stockage dans une base de données est également interdit. 


\title{
L'approche ressources et compétences comme clé de lecture du processus d'élaboration d'une ressource originale : la marque territoire
}

\author{
Corinne ROCHETTE ${ }^{1}$
}

\begin{abstract}
Résumé ${ }^{1}$
La concurrence grandissante entre les territoires conduit les collectivités à se doter de marques et à adopter une démarche marketing. Cela se traduit par une évolution des compétences que permet d'appréhender l'approche par les ressources. Ses transformations sont étudiées à partir de la marque région " Auvergne Nouveau Monde ».

$\begin{array}{lr}\text { Mots clés: } & \text { Approche } \\ \text { ressources/compétences, } & \text { avantage } \\ \text { compétitif, processus collaboratif, marque } & \\ \text { territoire, marketing territorial } & \end{array}$
\end{abstract}

The resource-based view as a grid to read the process of the creation of an original resource: the brand of territory

\begin{abstract}
:
Because of the growing competitive context between territories, the public institutions adopt brands and a marketing approach. This phenomenon is linked with an evolution of the skills; it is studied here by using the resourcebased view and through the example of the brand "Auvergne Nouveau Monde ".

Key-words: resource-based view, competitive advantage, collaborative process, brand of territory, territorial marketing
\end{abstract}

${ }^{1}$ CRCGM Clermont Université, pôle tertiaire, 26 Avenue Léon Blum, 63000 Clermont Ferrand; LARGEPA Paris 2, 1, rue Guy de La Brosse, 75005 Paris 2, corinne.rochette@udamail.fr, Maître de conférences en sciences de gestion.

\section{Introduction}

Les collectivités territoriales ont longtemps rejeté la possibilité de recours au marketing considéré comme incohérent avec leur mission de service public. Aujourd'hui, certaines investissent le champ du marketing stratégique et développent des marques région. Une marque territoire n'est pas une marque commerciale comme les autres. D'abord parce qu'elle est avant tout attachée à un espace physique, un territoire, et ensuite parce qu'elle réunit des offres très variées: une offre touristique, une offre de formation et avec ses écoles et ses universités, une offre de santé avec ses hôpitaux, une offre économique avec ses industries, ses pôles de compétitivité, un potentiel d'innovation et scientifique avec ses laboratoires de recherche..., mais aussi une qualité de vie, une culture, un mode de vie. Le territoire, constitué de nombreuses ressources tangibles et intangibles est aussi composé d'une multitude d'acteurs qui s'approprient le sens et les atouts dont il est porteur. Le nom du territoire, est utilisé par ceux désireux d'affirmer leur identité comme élément de valorisation et de différenciation. Chacun cherche alors à tirer avantage d'un toponyme apposé sur une offre sans qu'il y ait une mise en cohérence des actions de ces acteurs dans leur ensemble. 
Les régions, pour corriger leur manque de lisibilité au-delà des frontières nationales et homogénéiser l'usage de ce patrimoine collectif qu'est le toponyme, se dotent de ce nouvel outil qu'est la marque région. On peut d'abord s'interroger sur la nature même de cette marque: constitue-t-elle une ressource au même titre que les marques commerciales? Sa création et son développement nécessitent pour les collectivités d'intégrer une certaine logique marketing (bien différente de celle de service public), de se familiariser avec de nouveaux outils et démarches. Comment les collectivités font-elles évoluer leurs compétences ? Quelles sont les nouvelles compétences qu'elles acquièrent à travers ce projet de marque ? L'analyse et la compréhension de ce phénomène émergent ouvre de nombreuses voies de recherche. II en est une qui offre un cadre conceptuel robuste, largement validé dans le champ du management stratégique par les études et les recherches conduites sur les avantages compétitifs développés par les entreprises: il s'agit de l'approche par les ressources (Resource-Based View). Cette approche n'a pas été exploitée pour analyser le phénomène de marque territoire. Cet article se propose de mobiliser l'approche par les ressources (RBV) pour comprendre le processus d'élaboration d'une ressource originale qu'est une marque territoire et de voir en quoi celle-ci peut permettre aux collectivités de développer un avantage compétitif et de faire évoluer leurs compétences en particulier dans le domaine du marketing et de la stratégie.

Il convient de commencer par exposer dans une première partie les enjeux du marketing territorial, de préciser l'intérêt d'apposer sur un territoire une marque avant que de discuter de la transposition du modèle RBV (utilisé pour comprendre l'avantage compétitif développé par les firmes) au territoire. Une fois le cadre conceptuel mis en place, les propositions de recherche, le cadre méthodologique ainsi que les résultats de l'étude exploratoire sont présentés et discutés dans une deuxième partie. La compréhension de ce phénomène émergent de marque territoire à partir de l'approche par les ressources est réalisée par l'intermédiaire de l'étude du cas de la marque région " Auvergne Nouveau Monde ».

\section{La valorisation des ressources, marques et stratégies territoriales}

Dans un contexte de concurrence renforcée entre les territoires, l'attractivité devient une préoccupation majeure des collectivités. Le territoire peut être envisagé comme un produit à vendre nécessitant l'adoption d'un marketing adapté, le marketing territorial. La marque apposée sur un territoire conduit à s'interroger sur sa nature même de ressource et à envisager son développement comme une nouvelle compétence.

\subsection{Le développement de stratégies territoriales fondées sur les marques}

\subsection{1.}

\section{Une priorité pour les territoires : I'attractivité}

Le territoire (pays, régions, départements, villes) défini par des caractéristiques physiques (relief, climat), sociales, culturelles, économiques, politiques, administratives mais aussi symboliques et sensorielles est désormais soumis à une véritable concurrence, chacun s'évertuant à attirer touristes, étudiants, chercheurs, porteurs de projets.... II est la composante identitaire d'un espace (Levy et Lussault 2003) que s'approprient et mobilisent les acteurs (institutions, entreprises ...) pour exister sur divers marchés. A I'heure des Dircoms, les politiques de communication des collectivités territoriales par l'intermédiaire de discours accessibles sur de multiples supports s'attachent à créer cette identité qui permet d'exister ou d'en corriger une mal vécue. 
Chacune cherche à se différencier, à devenir ou rester attractive. "La décentralisation a largement contribué à instaurer une "logique marketing " dans laquelle le toponyme est devenu un élément clé de l'attractivité du territoire " (Boyer et Cardy 2011).

Les territoires font du renforcement de l'attractivité une priorité et mettent de plus en plus en place de véritables stratégies, parmi celles-ci le développement récent de marques territoire semble tenir une place de choix. Le nom, de simple vecteur de communication utilisé dans une perspective opérationnelle, devient un élément d'orientation stratégique guidant les actions et politiques publiques territoriales. On est passé d'un usage passif du nom à un usage actif, voire proactif. La mondialisation exacerbe la concurrence entre les territoires qui entrent en concurrence pour capter les facteurs mobiles que sont les facteurs de production, le capital, le savoir faire et donc les entreprises, les actifs, les chercheurs. Le territoire, lui, est un facteur fixe aux caractéristiques données (géographiques, climatiques, naturelles...) qui pour attirer les facteurs mobiles est contraint de créer des "facteurs visqueux", c'est-à-dire de mettre en œuvre des politiques territoriales d'attractivité destinées à fixer les facteurs mobiles. Le marketing territorial s'inscrit dans cette perspective là.

\subsubsection{Le marketing territorial: un outil au service de l'attractivité}

Né véritablement dans les années 1980, il est au départ essentiellement abordé dans sa dimension opérationnelle, celle de la communication. Après une certaine éclipse au cours des années 1990, l'intérêt pour le marketing territorial rejaillit. Pour Jarreau (1988) il s'applique plutôt au marketing des départements et des régions, celui appliqué aux villes étant qualifié de marketing urbain. Les travaux portant sur le marketing des territoires se sont concentrés sur le marketing des villes. Dès le départ il se définit comme un marketing d'image dont les composantes principales sont la promotion et la communication. Il continue à susciter de nombreux débats académiques en particulier sur la transposition des concepts du marketing de grande consommation, de ses démarches et outils au territoire. Les travaux jusqu'alors conduits traitent des limites de cette transposition et interrogent sur la nature même de ce marketing. Si la définition du concept de marketing territorial reste inachevée, les débats ont contribué à faire évoluer le concept et à l'enrichir de nouvelles dimensions. On cerne désormais un peu mieux quel est ce "produit » à promouvoir et/ou à « vendre " qu'est le territoire. Quoi qu'il en soit il s'impose de plus en plus comme une solution pour les collectivités désireuses de renforcer leur présence sur les marchés et sur la scène européenne voire internationale.

\subsubsection{Le territoire un "produit " singulier}

Si au départ pour certains (Bouinot et Bermils 1993; Guet et Pierre 1993) le marketing territorial est similaire au marketing de grande consommation dont il n'est qu'une déclinaison particulière, les débats et travaux auxquels il donne lieu montrent qu'il ne peut visiblement pas être réduit à la simple réplication des outils et démarches du marketing de grande consommation à cet objet particulier qu'est le territoire, en raison d'une part de l'importance du facteur politique (Sperling 1991) et d'autre part parce qu'un territoire n'est pas un produit comme un autre. Cette évolution s'explique probablement par un élargissement du champ de l'offre couverte par le marketing territorial. En effet, dans les années 1960, les actions marketing développées par les collectivités concernent le secteur du tourisme, la politique d'image est alors découplée de l'offre. L'image est prise en charge par les 
collectivités (territoires) alors que la politique de produits et donc de marque est prise en charge par les opérateurs du marché, du tourisme, qui développent des produits touristiques (séjours). Aujourd'hui, les cibles se sont élargies, les territoires ne s'intéressent plus uniquement aux touristes. Pour toucher et attirer leurs nouvelles cibles (entreprises, actifs, étudiants...), ils mettent en avant une offre bien plus large dont les composantes sont liées à l'action publique. L'évolution et l'adaptation de l'offre aux attentes des cibles (transport public, qualité de soins...) exigent des investissements lourds et du temps. En cela le territoire présente une réactivité bien différente de celle de l'entreprise à l'adaptation du produit à la demande (MargotDuclos 2011). Par ailleurs, la marque qui est un élément de valorisation de l'offre, une promesse faite au client, constitue pour les collectivités (plus particulièrement pour les régions) un moyen pour :

- renforcer l'attractivité du territoire ;

- se différencier des autres régions ;

- exister au delà les frontières régionales ;

- rendre lisible les actions (dont celles politiques) ;

- rassembler l'ensemble des acteurs du territoire autour d'un projet partagé ;

- mettre en cohérence les offres territoriales.

Comme en attestent les objectifs énoncés cidessus par les régions s'étant lancées dans un projet de marque territoire, nous pouvons noter que les ambitions associées à la marque sont fortes.

\subsection{4. \\ La marque territoire : pourquoi faire?}

La marque (de manière générale tous les éléments de marquage) exprime sa puissance sur deux niveaux : à l'externe dans la création $d$ 'une valeur supplémentaire en débanalisant l'offre et en interne en tant que facteur de mobilisation. Elle a, à la fois une fonction défensive de différenciation d'un produit de celui de la concurrence et une fonction symbolique, identitaire, d'incarnation d'une promesse (Kapferer 2011a). Elle est créatrice de valeur financière et constitue en cela une ressource importante (le "made in France" dans le domaine du luxe par exemple) mais elle est aussi un processus de mobilisation de tous derrière un projet commun s'exprimant à travers des valeurs. Ainsi toute politique de marque a pour premier enjeu d'assurer le contrat de base de confiance, car une marque sert d'abord à rassurer. "La politique de marque commence en interne au niveau de la production et des ressources humaines" (Kapferer 2011b), elle crée un engagement. «La marque territoire porte un projet de société, des éléments intangibles, des leviers d'attractivité symbolique ". Une marque territoire est donc avant tout un outil identitaire dont les racines sont bien plus profondes que celles d'une marque commerciale, elle est un condensé de projet de territoire, elle investit le champ de I'identité territoriale. Si la marque territoire en elle-même ne crée pas de valeur (et contribue bien peu à l'image), le processus d'appropriation et de valorisation par les acteurs d'un territoire est, lui, générateur de valeur. Le tableau 1 reprend les différences majeures entre une marque "territoire" et une marque " produit ». 
Tableau 1 : Les principales spécificités des marques "territoire" par rapport aux marques " produit "

\begin{tabular}{|c|c|}
\hline Marque " produit » & Marque « territoire » \\
\hline $\begin{array}{l}\text { - Fonction d'identification } \\
\text { - Fonction de garantie } \\
\text { - Fonction de valorisation du produit }\end{array}$ & $\begin{array}{l}\text { - Fonction politique de valorisation des } \\
\text { actions des élus } \\
\text { - Rendre visible (et lisible ?) un territoire } \\
\text { - Fonction de mise en cohérence des } \\
\text { " offres" }\end{array}$ \\
\hline - Création d'une identité & - Exploitation d'une identité \\
\hline - Offre concernée homogène & - Offre concernée hétérogène \\
\hline $\begin{array}{l}\text { - Cibles restreintes (marque/segment } \\
\text { et/ou cible) }\end{array}$ & - Cibles très larges \\
\hline - Marque excluante & - Marque englobante \\
\hline - Peut être vendue & - Ne peut être vendue ou cédée \\
\hline $\begin{array}{lll} & \text { Le produit un facteur aux } \\
\text { caractéristiques adaptables }\end{array}$ & $\begin{array}{llll} & \text { Le " produit » un facteur aux } \\
\text { caractéristiques fixes, figées } & & \end{array}$ \\
\hline
\end{tabular}

Dans son article de 2011, MargotDuclos s'appuyant sur une analyse des nombreux classements internationaux rendant compte des performances des territoires sur divers critères montre qu'aucun des classements ne fait référence à la marque et conclut qu' "il n'existerait pas de demande mondiale de marque de territoire, en un mot, pas de marché ". Ces classements sont à la fois des vecteurs et des révélateurs d'image, ils ont un impact sur les politiques mises en œuvre sur les territoires. Ils conduisent les collectivités à orienter les politiques publiques et à modifier les pratiques pour être " médaillables". La marque serait donc plus un outil de mise en cohérence de l'offre territoriale et de création d'un consensus entre les acteurs d'un territoire sur un projet commun qu'un outil de promotion externe. Cette perspective mérite d'être validée par une identification des enjeux et des pratiques de développement d'une marque territoriale.

Source : Création de l'auteur

L'adoption et le développement de marques territoire s'inscrit indubitablement dans une approche stratégique. Les termes d'attractivité, de compétitivité, de différenciation empruntés au champ de la stratégie d'entreprise leur sont largement associés dans la littérature. La marque territoire devrait contribuer au développement d'un avantage compétitif (synonyme d'attractivité) comme le soulignent les porteurs de ces projets de marque (pour la plupart institutionnels) cependant aucune recherche ne l'a validé et peut permettre de l'affirmer.

\subsection{Le territoire vu comme un ensemble de ressources, sa valorisation comme une compétence}

L'approche par les ressources (resource-based view) a apporté au management stratégique au milieu des années 1980 un cadre théorique abondamment mobilisé depuis plus de vingt ans pour comprendre comment un avantage 
compétitif est obtenu et peut être maintenu dans le temps par l'entreprise (Penrose 1959 ; Wernerfelt 1984 ; Prahalad et Hamel 1990 ; Barney 1991 ; Nelson 1991 ; Peteraf 1993 ; Teece, Pisano et Shuen 1997).

\subsubsection{Du facteur de production au concept de ressource}

Le concept traditionnel de stratégie peut être résumé en termes de position par rapport aux ressources c'est-à-dire de forces et de faiblesses (Andrews 1971). Les travaux de Penrose dans les années 1950 abordant l'entreprise comme un ensemble de ressources (1959) n'ont eu que peu d'échos. Jusque dans les années 1970 la principale caractéristique associée aux ressources est celle des rendements décroissants. Ne sont alors considérés que les facteurs de production (domination de l'approche néoclassique) élastiques, ajustables à court terme (Barney 2001) pouvant être achetés et vendus. Ainsi, par exemple, les compétences technologiques ne sont pas considérées comme un facteur de production et ne font pas l'objet d'un traitement. Une logique qui s'applique bien peu au territoire constitué de nombreux facteurs intangibles (son histoire, sa culture, son climat,..) et/ou de facteurs non mobiles (son patrimoine, sa géographie,...). L'idée que l'entreprise peut tirer bénéfice de liens non financiers est alors largement délaissée par les économistes. L'approche par les ressources va permettre d'ouvrir de nouvelles voies de recherche en distinguant le facteur de production de la ressource qui, elle, apporte un service (Penrose 1959). La RBV permet de comprendre la différence de performance entre les entreprises (création et maintien d'un avantage concurrentiel) à partir de l'usage fait des ressources. En effet, l'avantage concurrentiel tel qu'il est abordé par Porter ne réside alors plus nécessairement dans l'exploitation d'une position dominante et protégée sur un marché mais dans une valorisation supérieure des ressources (Tywoniak 1998). L'article fondateur de
Wernerfelt en 1984 propose une définition générale de la ressource sur la base d'une distinction entre les actifs tangibles et intangibles associés de manière semipermanente à l'entreprise. Ses apports sont conséquents. Ainsi la marque, l'image, les contrats commerciaux, les procédures, les connaissances technologiques... sont des ressources intangibles désormais intégrées dans l'analyse de l'origine de l'avantage compétitif. Certaines ressources telles que les compétences comportent un intérêt majeur dans la mesure où elles sont le résultat de l'accumulation des connaissances.

Les travaux conduits sur la nature de la ressource permettent d'en dégager les spécificités. Parmi celles-ci on retiendra que certaines d'entre elles ne peuvent pas être vendues ou achetées, que leur valeur peut être déterminée par le contexte, que l'utilisation de la ressource par plusieurs acteurs permet d'en accroitre la valeur et la profitabilité. Ces spécificités sont parfaitement applicables aux composantes d'un territoire. À l'échelle d'un territoire, le patrimoine, I'histoire et la culture ne peuvent pas être cédés à un autre. Un territoire sauvage peut être considéré comme une ressource pour le marché du tourisme mais comme un handicap majeur pour celui de l'entreprise dont l'implantation sur un territoire est conditionnée à la présence et à la densité des infrastructures. Quant à la marque territoriale, elle est une ressource intangible à rendement croissant. Plus elle est utilisée, plus elle contribue à la différenciation (positionnement ou repositionnement), à la notoriété, à l'image donc plus elle génère de rendements. La différence de performance en termes d'avantage concurrentiel s'explique par les différences de ressources et surtout par l'usage qui est fait de celles-ci. Barney (1991, 2002) définit quatre caractéristiques fondamentales (modèle VRIN) que doivent avoir les ressources pour que l'entreprise puisse conserver un avantage concurrentiel. Elles doivent être de valeur c'est-à-dire 
permettre de répondre aux opportunités du marché, être rares, inimitables et non substituables. A ces caractéristiques peuvent être rajoutées la longévité et l'appropriation. La première, la longévité, permet de maintenir l'avantage compétitif dans la durée et s'avère plus liée à la compétence qu'à la ressource (Grant 1991), bien qu'elle soit aussi déterminée par des éléments environnementaux (présence de nouveaux entrants, apparition de substituts). La seconde, l'appropriation, fait référence à l'organisation des processus et de la structure pour pouvoir exploiter pleinement la ressource (Barney 1995).

Les ressources singulières $d$ 'un territoire que sont le patrimoine architectural, gastronomique, les paysages, l'identité... comportent ces caractéristiques, elles sont rares, inimitables, non substituables, en revanche leur valeur dépend de la capacité des acteurs d'un territoire à organiser l'exploitation de ces ressources aujourd'hui valorisées par des consommateurs en mal d'authenticité. Des recherches ont montré que les entreprises qui construisent leur stratégie sur des éléments socialement complexes, ambigus, intangibles sont plus performantes que celles qui construisent leur stratégie sur des éléments tangibles (Makadok 2001), elles disposent alors d'une compétence particulière. Le processus collaboratif qui est une particularité du fonctionnement des territoires autour de projets collectifs présente ces dimensions de complexité sociale et d'ambigüité qui le rendent difficilement accessible et réplicable. Par ailleurs, la croissance optimale de l'entreprise implique un arbitrage entre l'exploitation de ressources existantes et le développement de nouvelles (Penrose 1959; Rubin 1973; Wernerfelt 1977). Les collectivités s'attachent, à travers les politiques mises en œuvre, à valoriser leurs ressources existantes mais aussi à en développer de nouvelles (la construction d'un aéroport, d'une scène nationale de spectacles, d'un centre de congrès, la création d'un cluster, la labellisation d'un pôle de compétitivité... mais aussi la création d'une marque). Une démarche qui contribue à la construction d'une image et d'un avantage compétitif grâce à l'élaboration d'un système d'offres et de prestations reposant sur les ressources détenues. Le développement de nouvelles combinaisons de ressources relève de la compétence, il permet de créer plus de valeur.

\subsubsection{Le développement d'une marque territoire vu comme une nouvelle compétence}

Dès la fin des années 1990 les chercheurs ont intégré à l'approche RBV une dimension dynamique fournie par l'approche par les compétences de Prahalad et Hamel (1990) en développant des modèles évolutionnistes. Ils (Teece, Pisano et Shuen 1997 ; Karim et Michell 2000) s'intéressent alors à la façon dont sont exploitées les compétences de l'entreprise (théories des compétences) et à la façon dont elles évoluent avec le temps (dynamic capabilities) ainsi qu'aux implications stratégiques de ces changements. L'entreprise est appréhendée comme un ensemble de compétences organisées en une structure (Quelin 1995; Grant 1996). II revient à l'organisation de réaliser l'intégration des savoirs fragmentés de chaque membre en en facilitant l'utilisation et le partage. Cette compétence organisationnelle n'est pas donnée à toutes les organisations, mais celles qui en disposent valorisent mieux leurs ressources. Quelin (1995) distingue trois niveaux d'intégration des compétences. Le premier, le niveau élémentaire concerne des compétences liées à des actions opérationnelles, le deuxième, le niveau intermédiaire renvoie à des compétences fonctionnelles enfin le troisième, dit supérieur, est relatif aux compétences inter-fonctionnelles et générales. Le développement d'une marque territoire implique d'abord une assimilation de la logique marketing, la mise en œuvre de nouvelles pratiques et l'usage de nouveaux 


\section{L'approche ressources et compétences comme clé de lecture du processus d'élaboration d'une ressource originale : la marque territoire/C Rochette}

outils dont les collectivités ne sont pas familières, en résumé elle va de pair avec le développement de nouvelles compétences (marketing et stratégique). Le succès du projet de marque territoire est probablement conditionné à la présence de compétences particulières sur ces trois niveaux. La création d'une marque territoriale exige des compétences inter-fonctionnelles et générales. En effet, pour donner vie à une marque partagée, il convient de mobiliser de très nombreux acteurs (entreprises, collectivités...) dotés de cultures métier très différentes (langages, pratiques) de les faire adhérer et travailler sur un projet commun. Elle nécessite, ensuite, d'appliquer une démarche marketing pour identifier le positionnement de la marque, de bâtir des plans de communication c'est-à-dire d'acquérir des compétences fonctionnelles (niveau intermédiaire), assez lointaines de celles traditionnellement associées aux collectivités qui ne disposent d'ailleurs pas de service marketing. Enfin sur le plan opérationnel (niveau élémentaire), la création de la marque va de pair avec des compétences en matière de conception d'outils de communication (site internet, plaquettes...), de réalisation d'études (étude d'image, de notoriété). Ainsi, l'enjeu pour les porteurs du projet de marque territoire est d'intégrer de nouvelles compétences. Les interactions sociales sont au cœur de ce processus d'apprentissage. Les collectivités peuvent avoir recours au benchmarking et ainsi apprendre de villes, départements, régions dont le projet est plus avancé ou achevé, mais aussi d'entreprises, mobiliser des experts (société de conseil, universitaires). La création de connaissances nouvelles repose sur une fertilisation croisée et la compétence intégrative de l'organisation. Collis (1991) montre combien parfois "l'héritage administratif » peut expliquer le décalage entre les évolutions structurelles observées (ce qui est) et les prescriptions (ce qui devrait être). La culture de service public des collectivités peut être un frein à l'acquisition de nouvelles compétences. Dans un environnement marqué par des évolutions rapides, la capacité à intégrer, construire et reconfigurer les compétences internes et externes est déterminante pour obtenir un avantage compétitif (Teece et al. 1997). La capacité à absorber et appliquer des connaissances nouvelles repose soit sur l'exploration soit sur l'exploitation (Wright, Van Wijk et Bouty 1995). Dans le premier cas le savoir est théorisé, codifié avant d'être utilisé, dans le deuxième cas il est intégré et utilisé empiriquement avant d'être codifié. Les organisations favorisent l'exploration ont tendance à être plus innovantes mais peuvent rencontrer des difficultés dans l'exploitation des nouvelles compétences. II semblerait que ce soit le cas pour les collectivités qui développent des compétences de nature marketing. Makadok (2001) parle de théories de la construction de compétences (capability building theories). Elles permettent, en particulier, de comprendre comment se créent de nouvelles ressources. Les capacités dynamiques consistent en des processus organisationnels et stratégiques spécifiques qui créent de la valeur. Eisenhardt et Martin (2000) les associent étroitement à la notion de routines et montrent combien le rôle joué par les capacités dynamiques dans l'acquisition d'un avantage compétitif est lié aux caractéristiques de stabilité ou d'instabilité de l'environnement. Ainsi dans un environnement fortement évolutif, les routines ne permettent pas de développer un avantage, les capacités dynamiques qui consistent en des processus simples, changeants, non linéaires, peu structurés permettent, elles, de développer un avantage compétitif dont l'origine n'est pas seulement exogène (opportunité de marché par exemple) mais endogène et donc plus durable. L'avantage compétitif obtenu à partir des capacités dynamiques semble plus naturellement fondé sur la différenciation, il permet de créer un surplus de valeur, les routines contribuant, elles, à développer des avantages plus fondés sur les coûts. 
L'approche par les ressources et compétences a été peu utilisée dans le domaine du marketing territorial, elle peut aider à expliquer la façon dont les collectivités construisent et reconfigurent leur stratégie de marque et dont elles s'adaptent aux évolutions de l'environnement (décentralisation, concurrence entre territoire du fait de la mondialisation) et font évoluer leur compétence (application, appropriation voire création de démarches et outils marketing jusqu'alors ignorés). L'environnement dans lequel celles-ci évoluent est changeant, l'approche par les capacités dynamiques fournit une grille de compréhension de la façon dont les activités stratégiques des collectivités contribuent au développement de nouvelles configurations de ressources (Eisenhardt et Martin 2000). La marque est un instrument (une nouvelle ressource) pouvant permettre de construire, obtenir ou renforcer un avantage compétitif (Abimbola 2001). Le développement d'une stratégie de marque pour reconfigurer les ressources et capacités dans un environnement turbulent peut être appréhendé comme une capacité stratégique (Matanda et Ewing 2012).

$\mathrm{Si}$ la marque est une ressource intangible son processus de développement et de gestion est une compétence; aussi, la théorie des capacités dynamiques fournit-elle un cadre théorique idéal pour étudier et expliquer le développement de marque territoire.

\section{Le cas exemplaire de la marque " Auvergne Nouveau Monde "}

\subsection{Propositions de recherche et méthodologie}

Nous nous situons ici au niveau de l'entité région. Les régions sont un terrain particulièrement riche pour étudier la façon dont se construisent ces nouvelles pratiques et se développent de nouvelles compétences de nature plus marketing et stratégique. Elles affichent clairement leurs ambitions : exister, se démarquer, renforcer leur attractivité, des objectifs au service desquels se trouvent les marques région. Trois régions françaises se sont aujourd'hui dotées d'une marque régionale. La Bretagne a été la première à lancer sa marque en 2008, l'Auvergne en a fait de même en 2010, en 2012 ce fut à l'Alsace de se doter de cet outil. Ces trois régions se caractérisent par une identité forte et très marquée. II s'agit probablement d'un prérequis (une ressource intangible conditionnelle) à la création et au déploiement de la marque. Le terrain choisi est celui du cas de la marque "Auvergne Nouveau Monde ».

Le recueil de données c'est fait en deux temps.

- Une collecte documentaire (schéma régional d'aménagement du territoire, rapports d'identité, délibérations et compte rendus des séances de l'agence régionale de développement des territoires d'Auvergne, études du comité régional du tourisme, brochures, articles de presse) traitée grâce au logiciel d'analyse discursive TROPES 8.0 (encadré 1 ), afin de repérer les thèmes clés associés à la marque région et les acteurs à interroger. Le comité du tourisme, le conseil régional ont été identifiés comme étant les deux piliers du projet.

- La deuxième étape s'est déroulée autour d'une série d'entretiens semidirectifs d'une durée de 45 minutes à 1 heure 30 auprès d'un directeur général des services du conseil régional, du directeur du comité régional du tourisme en charge de la marque, du directeur de la prospective, du responsable du service de développement des territoires, de la personne en charge du déploiement de la marque pour I'association «Auvergne Nouveau 
Monde ». Les entretiens ont été enregistrés et intégralement retranscrits. Les propos des acteurs ont été analysés à l'aide du logiciel TROPES 8.0 dont les résultats ont servi de guide à l'analyse thématique et lexicale manuelle.

\section{Encadré 1 : Le logiciel utilisé}

Tropes est un logiciel mis au point par un groupe de chercheurs sur la parole. Il connaît une large diffusion dans la communauté académique et auprès des praticiens. II autorise une analyse de verbatim afin que le résultat reflète, autant que possible, les verbatim originaux, évitant de fait, des biais analytiques hasardeux de la part du chercheur. Tropes 8.0 permet à la fois une automatisation des résultats et une dissociation entre les résultats obtenus et leur interprétation. Bâti autour d'un moteur de recherche sémantique et d'analyse documentaire, il autorise une analyse fondée sur la compréhension du contenu à traiter, et offre quelques fonctionnalités remarquables comme : la détection du contexte du texte, la catégorisation des mots-outils et leur appariement, la localisation de séries temporelles dans le discours. Chaque mot significatif est inscrit dans une chaîne d'équivalents sémantiques : un mot suffit pour retrouver tous les documents le contenant, lui et ses équivalents (synonymes). Par exemple, une recherche sur le mot " commerce " retrouvera les documents contenant les mots " marché ", " magasin " et " revendeur ", même si le mot " commerce " ne se trouve pas explicitement dans le texte.

L'analyse des données discursives a pour objectif d'évaluer la consistance des quatre propositions principales de recherche dégagées à l'issue de la revue de la littérature. Elles constituent l'armature de la grille d'analyse des données collectées.

Les quatre propositions ont été ainsi formulées :
- Proposition 1 : Les territoires cherchent à renforcer leur avantage concurrentiel ${ }^{2}$ la marque territoire est considérée comme un élément y contribuant par la différenciation.

- Proposition 2: La marque territoire est une ressource intangible créatrice de valeur.

- Proposition 3: La création et le développement d'une marque territoire est une compétence nouvelle.

- Proposition 4: La marque territoire est une nouvelle combinaison de ressources reposant plus sur les capacités dynamiques que sur des routines.

Ces quatre propositions doivent permettre d'évaluer la pertinence de l'approche RBV pour analyser le processus d'élaboration d'une ressource originale, la marque région.

\subsection{Les résultats et discussion}

\subsubsection{L'analyse des données secondaires}

Nous présentons tout d'abord l'analyse des documents collectés. Le traitement logiciel d'analyse textuelle nous apporte une information utile quand à la façon dont est abordé le projet de marque région et les enjeux qui lui sont associés. L'analyse statistique des données secondaires porte sur 19883 mots et 1577 propositions. 52,9\% des verbes utilisés sont factifs, ils expriment des actions plus que des états $(29,2 \%$ de verbes statifs), les documents ayant pour objectif de

\footnotetext{
${ }^{2}$ Notons que la notion d'attractivité est, pour les collectivités, équivalente à l'expression d'avantage concurrentiel, une différence sémantique liée à une représentation très différente de la concurrence entre le secteur privé-marchand et celui public.
} 
présenter les actions envisagées. L'importance $(43,9 \%)$ des adverbes d'intensité détectée dans les textes et leur nature soulignent l'intensité donnée aux propos. On relève une forte proportion d'adjectifs numériques $(48,7 \%)$ et objectifs $(30,1 \%)$. Les documents émanent d'institutions, aussi ces dernières étayent et justifient le projet à partir de données chiffrées (diagnostic de flux de population, budget, évolution...), ce qui lui donne plus de force et le rationalise. Les dix références les plus utilisées dans les supports mettent en évidence les points d'ancrage de la marque Auvergne, parmi elles, identité, qualité, terre. La présence forte de "Puy de Dôme " (classe constituée du département et de la capitale régionale, Clermont Ferrand) suggère que l'initiative provient du cœur géographique et administratif de la région et met en relief son caractère centralisateur, même si les autres départements sont cités. Ceci suggère une forme de confiscation du projet.

Les dix références les plus employées pour évoquer la marque Auvergne renvoient à la nécessité de construire une politique de marque pour l'Auvergne, et ce en raison de I'hyper concurrence à laquelle sont soumis les territoires et de la nécessité de renforcer l'attractivité. L'analyse logicielle offre la possibilité d'identifier les associations de mots. Leur récurrence rend compte de la force $\mathrm{du}$ lien entre deux termes. Parmi les associations les plus fortes, on trouve assez naturellement "marque/Auvergne" (39) et "stratégie/marque " (21). La marque relève donc de la stratégie. II s'agit principalement d'une stratégie de communication. Les termes "positionnement ", " différenciation ", " avantage " couramment associés à la stratégie de marque commerciale ne sont pas détectés. La conception de la marque région semble être différente de celle des marques de produits ou de services "classiques». La place des hommes dans le projet apparaît clairement. Une lecture des supports a permis de voir l'importance de la préoccupation démographique, la volonté de faire de la marque Auvergne une marque destinée à attirer de nouvelles populations (habitants, touristes, entreprises, étudiants). Elle est aussi un projet collectif, un élément rassembleur, dans lequel chacun doit se reconnaître. Elle est un outil pour accroître l'attractivité du territoire. L'Auvergne conserve un déficit d'attractivité pour des raisons d'image perçue.

Cette première étape dans l'analyse nous conduit à constater que le territoire est traité dans ses dimensions symbolique et identitaire. La composition culturelle de la marque est nette. Le territoire auvergnat peut effectivement être envisagé comme une marque collective co-construite destinée à supporter les politiques d'attractivité, elle constitue en cela une véritable ressource. Cependant même si la volonté de mobiliser les acteurs autour du projet apparaît nettement, le projet est bien l'émanation d'une autorité centrale et est porté par elle. La dimension stratégique, bien que présente, semble plus être reliée à la communication. L'analyse de ces données secondaires ne permet pas de voir si le processus de fabrique de cette marque constitue une nouvelle compétence, et si la création de cette marque est le résultat d'une nouvelle combinaison de ressources, ce que devrait permettre de dégager l'analyse des propos des personnes qui travaillent à sa constitution.

\subsubsection{L'analyse des entretiens, les principaux résultats}

- En préambule, quelques chiffres...

Trois directeurs et deux responsables de service ont été interrogés aux mois d'avril et mai 2011 et mars 2012. Les propos recueillis représentent 3609 propositions et 32157 mots. Le style des discours est plutôt argumentatif pris en charge par le narrateur. Le logiciel a détecté des notions de doute. On constate un équilibre entre l'emploi de verbes statifs $(39,4 \%)$ révélateurs d'un état, d'une possession et ceux factifs $(40,2 \%)$ en lien avec l'action. A la différence du contenu des 
supports analysés précédemment, les propos comportent une proportion plus importante d'adjectifs subjectifs, $44,6 \%$ pour $40,5 \%$ d'adjectifs objectifs, ce qui rend compte de l'appropriation du discours par les personnes interrogées.

Parmi les 10 références les plus utilisées, on retrouve les références piliers que sont "Auvergne", " marque ", " région » et " territoire ». Les autres références apportent un éclairage nouveau. On voit surgir dans le discours les dimensions "politique», des dimensions temporelles (" année") et humaines ("gens ") de façon très marquée. On soulignera aussi la présence de la référence "chose " qui a son importance. En effet, celle-ci indique une difficulté à qualifier, ou nommer certains éléments relatifs au processus de création de la marque région probablement en raison de la nouveauté des concepts manipulés et de la difficulté pour des collectivités territoriales à appréhender de nouveaux outils et techniques, ceux du marketing.

\section{- La marque "Auvergne Nouveau Monde " une ressource stratégique portée par de nouvelles compétences}

La création et le développement de la marque territoire permettent de mettre en évidence l'acquisition de nouvelles compétences tant pour les porteurs du projet que pour les utilisateurs (porteurs) de la marque. II a fallu d'abord construire cette marque en mettant en résonnance les ressources (fixes tangibles et intangibles) dont dispose le territoire avec les opportunités qu'offre l'environnement puis en créant des synergies entre les acteurs du territoire contribuant à sa valorisation et à son image.

L'objectif de création de la marque a été résumé comme la volonté de "ne plus subir la marque $^{3}$ mais de la construire ". II s'agit donc

\footnotetext{
${ }^{3}$ Entendons ici par marque, une image négative.
}

bien là de mettre en œuvre des compétences. L'Auvergne souffre depuis de nombreuses années d'un déficit d'image (une région fermée, austère, méconnue), alors qu' "elle incarne plus que tout autre territoire la naturalité, la qualité et la pureté que tout le monde recherche aujourd'hui ". En effet, l'évolution de l'environnement marquée par l'émergence de nouvelles attentes et exigences des consommateurs constitue une véritable opportunité pour l'Auvergne qui dispose d'importantes ressources naturellement associées à ces caractéristiques et de faire de son "image de région austère, méconnue, préservée ") une véritable ressource. La capacité à mettre en avant des valeurs (longtemps délaissées) au bon moment, en en faisant un élément différenciant de l'offre territoriale contribue à renforcer l'attractivité du territoire et constitue une véritable compétence. II s'agit là d'une bonne illustration des capacités dynamiques de Teece, Pisano et Shuen (1997).

Par ailleurs, le choix sémantique n'est pas neutre car le langage construit le territoire. En adoptant le terme "nouveau» et en l'associant à Auvergne on oriente l'action de l'ensemble des acteurs. En effet, de prime abord ces deux termes paraissent antinomiques, l'image de l'Auvergne n'incarnant pas jusqu'à présent la modernité. "Quand on a nouveau dans son nom, on est obligé d'être innovant. On vous regarde sur votre capacité à innover à tous les points de vue ». Les propos rapportés ici montrent combien la marque et son positionnement orientent l'action. Une des fonctions attribuée à la marque "Auvergne Nouveau Monde » est d'encourager l'innovation, d'inciter les porteurs de cette marque à être novateurs donc à faire évoluer leurs pratiques et leurs stratégies propres. D'ailleurs, la création de la marque donne lieu à un cahier des charges (code de marque) que doivent respecter les acteurs désireux de l'utiliser. Parmi les éléments figurant dans le code de marque on trouve la nécessité de porter les valeurs de 
naturalité, d'exigence, d'idéalisme, de partage et de créativité du nouveau monde.

Les propos ont montré qu'il existait un risque de brouillage de l'image. En effet, lors des entrevues deux des personnes interrogées ont eu des difficultés à identifier la marque région. Plusieurs signatures coexistent encore : "Une région juste et grande " plutôt considérée comme étant une signature institutionnelle adoptée par le conseil régional, "l'effet volcan " utilisé par la région et le comité régional du tourisme pour communiquer et promouvoir les offres du territoire, "Bravo l'Auvergne" un label destiné essentiellement à des produits agroalimentaires. "Toutes ces signatures sont vouées à disparaître dans deux ou trois ans à condition que la marque "Auvergne nouveau monde " se soit imposée et prenne le relais." "La marque Auvergne Nouveau Monde est une marque collective dont la philosophie n'est pas sectorielle ", son déploiement nécessite l'implication et la mobilisation de tous: entreprises, collectivités, institutions, associations... car comme l'évoque Kapferer (2011b) "la politique de marque commence en interne au niveau de la production et des ressources humaines ". Ainsi, une des priorités des porteurs du projet (désormais l'association Auvergne Nouveau Monde) est d'accumuler des preuves pour soutenir la promesse faite en encourageant et en mettant en avant les pratiques et projets innovants. Ainsi le développement de la marque contribue à faire émerger de nouvelles combinaisons de ressources à deux niveaux :

- d'abord parce que chaque acteur porteur des valeurs de la marque en l'adoptant contribue à la renforcer, à la crédibiliser, à lui donner de la valeur. Plus elle sera adoptée, plus son rendement sera croissant. En effet, si au départ les départements étaient réticents à adopter cette nouvelle marque, ils y sont aujourd'hui plus ouverts (peut être contraints par nécessité ?) du fait du nombre importants d'utilisateurs. La marque contribue donc à créer des synergies fortes et à reconfigurer les relations entre les acteurs d'un territoire.

- Ensuite parce qu'elle contribue à homogénéiser les pratiques en termes de valorisation du territoire en portant l'ambition d'un territoire unanimement partagée.

Les premières étapes de la démarche concernent la réalisation de diagnostics très classiques reposant sur une démarche de type SWOT. Le diagnostic identitaire a permis de compléter les diagnostics socioéconomiques réalisés, de dégager collectivement un "positionnement autour de l'Auvergne, une région qui vous fait du bien " et de conduire une réflexion sur les valeurs associées à la région et celles auxquelles on souhaite l'associer pour l'avenir. II s'agit là d'une démarche totalement nouvelle pour une collectivité. En effet, si l'initiative de ce projet revient au Conseil régional et au Comité régional du tourisme, très rapidement le manque d'expertise les a conduits à se faire accompagner dans leur démarche, puis dans un deuxième temps à se réapproprier le projet en abandonnant toute assistance. En effet comme le soulignent deux des interviewés, l'accompagnement par des cabinets d'experts conduit à un formatage de la démarche (proche des routines), à la proposition de recettes qui sont finalement très similaires à celles appliquées par les autres régions dans la mesure où elles sont, elles aussi, accompagnées dans leur projet par les mêmes experts. Une situation qui limite finalement la portée de l'avantage compétitif apporté par la marque. À titre d'illustration le positionnement choisi par l'Alsace, "l'imagination" n'est pas très éloigné de celui de l'Auvergne, "le monde idéal». Le positionnement a été traduit en ce qui s'apparente à une mission générale "inventer une nouvelle... économie, façon de vivre, façon de se déplacer, façon de travailler... " qui fait office de feuille de route pour l'ensemble des acteurs et met au premier plan la créativité et 
l'inventivité. Des choix qui s'inscrivent dans un projet politique et qui trouvent déjà une formalisation institutionnelle à travers les groupes de réflexion appelés « manufactures » de la mission prospective du Conseil régional.

Une fois la marque créée, il s'agit maintenant de la faire vivre, de la développer. Les interviewés sont conscients des écueils, et des difficultés soulevées par la mise en œuvre des grandes lignes forces dégagées par la marque région. "Nous, les directions opérationnelles on met en ceuvre des actions avec les partenaires de chaque territoire pour renforcer l'attractivité de ces territoires et dont les préoccupations sont plus terre à terre» (entendons par territoires, les 22 pays qui constituent l'Auvergne gérés par des $\mathrm{EPCl}^{4}$, associations). Les acteurs institutionnels, que sont les départements, les pays et les villes sont réticents à abandonner leurs slogans et adhérer à cette uniformisation, ils craignent de perdre leur identité singulière.

En revanche les entreprises ont un rôle moteur à jouer: "Les entreprises ont compris tout l'intérêt qu'elles ont à utiliser cette marque", "l'identité auvergnate forte renforce le pouvoir de conviction", "le crédit mutuel va créer une carte Auvergne nouveau monde ", de même "la manufacture Michelin peut envisager d'associer la marque région à des produits innovants " ainsi que "la marque Volvic du groupe Danone ". II s'agit là de points clés qui vont permettre de donner de la lisibilité à la nouvelle marque. Les propos des interviewés soulignent aussi la nécessité de sélectionner les produits, services et projets en fonction de leur capacité à véhiculer les valeurs de la marque (" naturalité, qualité, pureté, créativité, inventivité »).

Le déploiement de la marque a été pensé en termes d'information et de communication. La première étape a été la

${ }^{4}$ Les établissements publics de coopération intercommunale présentation (en juillet 2011) aux habitants (appelés "pionniers") de "leur marque" dans un dossier de 16 pages dans le quotidien régional à forte audience et la création d'un site Internet dédié, puis en 2012 le lancement du magazine " Nouveau Monde ». La publicité dans les grands médias est abandonnée au profit du co-branding et les relations presse plus efficaces. La deuxième étape est l'appropriation de la marque par des portes drapeaux tels que les entreprises, les structures publiques et privées ... respectant les orientations définies par le territoire "nouveau monde» de la marque. La troisième étape amorcée dès 2012 est la mutualisation des moyens et l'harmonisation plus poussée des pratiques s'accompagnant de la disparition de certaines marques périphériques. En effet, la marque " Auvergne nouveau monde " est une marque qui se veut transversale sa diffusion devrait conduire à la disparition de marques que l'on pourrait qualifier de silos car trop sectorielles. Le souhait des porteurs de la marque est que "dans un avenir plus ou moins proche elle oriente l'action politique ".

\section{Conclusion}

La marque territoire constitue bien une ressource intangible créatrice de valeur d'abord non financière et à terme financière (la proposition 2 montre un fort degré d'acceptabilité). Le succès de sa création et de son déploiement repose sur une nouvelle combinaison de ressources en particulier intangibles (identité, image, relations entre les acteurs). Elle mêle dans un processus créatif l'identité et l'image du territoire avec l'identité et l'image des acteurs-utilisateurs. Elle contribue à faire évoluer les relations entre les parties prenantes à travers la création de nouveaux liens et le développement de liens de nouvelle nature. Sa création puis son déploiement impliquent l'acquisition de nouvelles compétences techniques à travers la mobilisation d'experts, mais aussi de compétences organisationnelles 
et relationnelles nées essentiellement de la pratique (les propositions 3 et 4 présentent un bon degré d'acceptabilité). Si l'avantage recherché dans le développement d'une marque région est l'attractivité, celle-ci ne peut pas être à ce jour considérée comme un synonyme d'avantage concurrentiel. En effet dans le discours des personnes interrogées la conscience de la concurrence est très faible, cependant elle est bien considérée comme un élément de différenciation de l'offre d'un territoire (la proposition 1 montre un niveau moyen d'acceptabilité).

Les apports académiques de cette recherche se situent dans la transposition de I'approche par les ressources à l'analyse d'une problématique territoriale. Le territoire a largement été étudié sous l'angle des facteurs de production mais la RBV fournit un cadre intéressant et aisément mobilisable pour analyser le processus de création d'une nouvelle ressource intangible: une marque territoire. Elle offre un moyen pour mieux appréhender l'évolution des compétences dans le domaine du marketing des collectivités territoriales, la diffusion et la construction de nouvelles pratiques. La marque territoire peut jouer un rôle dans la structuration et l'organisation des ressources d'un territoire en favorisant les interactions entre les acteurs publics et privés, elle permet de faire émerger de nouvelles combinaisons de ressources.

Les apports managériaux renvoient à la nécessité de dépasser le recours à des démarches formatées qui ne sont que des greffes qui peuvent peiner à se développer dans des secteurs où le marketing reste encore largement ignoré et le vocabulaire associé peu diffusé voir rejeté. Ainsi, par exemple, pour l'Auvergne le plan de développement de la marque s'est mué en schéma régional d'influence même si le contenu reste identique.
Les limites de ce travail se situent bien évidemment dans son caractère exploratoire et l'unicité du cas étudié qu'une duplication de l'étude permettrait de corriger. Par ailleurs, en mobilisant l'approche par les ressources on admet la nécessité d'introduire une perspective dynamique inscrite dans la mobilisation de l'approche par les ressources (capacités dynamiques). Aussi l'étude longitudinale de ce cas permettra d'intégrer cette nécessaire dimension.

\section{Bibliographie}

ABIMBOLA T., 2001, "Branding as a competitive strategy for demand management in SMEs", Journal of Research in Marketing and Entrepreneurship, 3, 2, pp. 97-106.

ANDREWS K. R., 1971, The concept of corporate strategy, Dow Jones-Irwin.

BARNEY, J. B., 1991, "Firm resources and sustained competitive advantage", Journal of Management, 17, pp. 99-120.

BARNEY, J. B., 2001, "Resources-based theory of competitive advantage: a ten years retrospective on the resource-based view", Journal of Management, 27, 6, pp.643-650.

BARNEY, J. B., 2002, Gaining and sustaining competitive advantage, Upper Saddle River, Prentice Hall.

BOUINOT B., BERMILS B., 1993, Projet de ville et projets d'entreprises, librairie générale de droit et jurisprudence, Paris.

BOYER H., CARDY H., 2011, "Localiser, identifier, Valoriser ", Mots. Les langages du politique, 97, pp. 5-13.

CHRISTMAS PP., 2000, "Effects of the "best practices" of environmental management on cost advantage: the role of complementary assets", Academy of Management Journal, 43, 4, pp.663-680.

COLLIS D.J., 1991, "A resource based analysis of global competition: the case of bearings industry", Strategic Management Journal, summer, pp.49-68. 
EISENHARDT K. M., MARTIN J. A., 2000, "Dynamic capabilities: what they are?" Strategic Management Journal, 21, 10/11, pp.1105-1121.

FLIPO J.PP., TEXIER L., 1992, " Marketing territorial de la pratique à la théorie " Revue Française de Marketing, 136, pp.41-52.

FREILING J., 2010, « Raison d'être et nature de la firme: les compétences comme socle explicatif ", Revue française de gestion, 204, pp.127-139.

GRANT R.M., 1996, «Prospering in Dynamically-competitive environments; organizational capability as knowledge integration», Organization Science, 4, Jul-Aug, pp.375-387.

GUETS J.F., PIERRE G., 1989, Marketing et stratégies urbaines, Fondation pour la gestion des villes et des collectivités locales, Paris.

JARREAU PP., 1988, "Survey sur l'image de marque des villes et le marketing territorial ", Cristal, Ministère de l'équipement et du logement, Direction de l'Architecture et de I'Urbanisme.

JEGO Y, 2010, « En finir avec la mondialisation anonyme, rapport à $M$. le Président de la république ", La Documentation Française,

KAPFERER J.N., 2011a, «Quelle stratégie pour la marque France demain ? ", Revue Française de Gestion, 218/219, pp.139-153.

KAPFERER J.N., 2011b, "France: Pourquoi penser marque?", Revue Française de Gestion, 218/219, pp.13-23.

KARIM S.Z., MITCHELL W., 2002, "Pathdependent and path-breaking change: reconfiguring business resources following acquisitions in the U.S. medical sector, 19781995, Strategic Management Journal, 21, 10/11, pp.1061-1081.

LEVY J., LUSSAULT M., (dir.), 2003, Dictionnaire de la géographie et de l'espace des sociétés, Saint-Just-la Pendue, Belin.
MAKADOK R., 2001, "Toward a synthesis of the resource-based and dynamic-capability views of rent situation", Strategic Management Journal, 22, 5, pp.387-401.

MANTADA T., EWING M. T., 2012, "The process of global brand strategy development and regional implementation", International Journal of Research in Marketing, 29, 1, pp.512.

MARGOT-DUCLOT J.L., 2011, "Paris et la France ", Revue Française de Gestion, 218219, pp.67-89.

NELSON R. R., 1991, "Why do the firms differ, and how does it matter?" Strategic Management Journal, Winter, special issue 12, pp.61-74.

PRAHALAD C., HAMEL G., 1990, "The Core Competence of the Corporation", Harvard Business Review, May-June, pp.79-91,

PENROSE E., 1959, The Theory of the Growth of the Firm, Oxford, Blackwell.

PETERAF M. A., 1993, "The cornerstones of competitive advantage", Strategic Management Journal, 14, 3, pp.179-191.

PORTER M., 1985, Competitive advantage: creating and sustaining superior performance, New York, The Free Press.

PREVOT F., BRULHART F., GUIEU G., MALTESE L., 2010, "Perspectives fondées sur les ressources ", Revue Française de Gestion, 204, pp.87-103.

QUELIN B., 1995, "Competence of the firm and strategic alliances: creation of new capabilities and appropriability", HEC, working paper, Sept.

RUBIN PPP. H., 1973, "The expansion of the firm ", Journal of Political Economy, 81, pp.936-949.

SPERLING D., 1991, Le marketing territorial, Toulouse, Milan-Midia. 
TEECE D.J., PISANO G., SHUEN A., 1997, "Dynamic capabilities and strategic management", Strategic Management Journal, 18, 7, pp.509-533.

TYWONIAK S.A., 1998, "Le modèle des ressources et compétences; un nouveau paradigme pour le management stratégique ? ", in LAROCHE H. NIOCHE J.P. (éd.), Repenser la stratégie - fondements et perspectives, Vuibert, 166-204.

WERNERFELT B., 1977, « An information based theory of microeconomics and its consequences of corporate strategy", Unpublished Dissertation, Havard University, graduate school of business administration.

WERNERFELT B., 1984, "A resource-based view of the firm ", Strategic Management Journal, 5, 2, pp.171-180.

WRIGHT R.W., VAN WIJK G., BOUTY I., 1995, "Les principes du management des ressources fondées sur le savoir", Revue Française de Gestion, 105, Sept-Oct., pp.7075. 